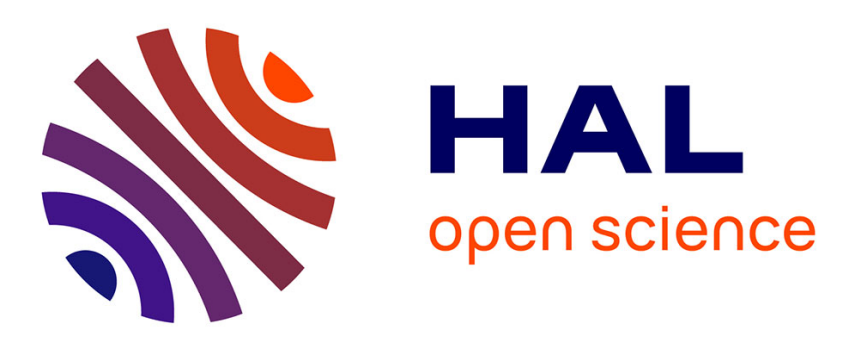

\title{
Unified mathematical model of the kinetics of nanoparticle phase condensation in magnetic fields
}

Pavel Kuzhir, Maxime Raboisson-michel, Jordy Queiros Campos, Gregory

Verger-dubois, Andrey Yu. Zubarev

\section{- To cite this version:}

Pavel Kuzhir, Maxime Raboisson-michel, Jordy Queiros Campos, Gregory Verger-dubois, Andrey Yu. Zubarev. Unified mathematical model of the kinetics of nanoparticle phase condensation in magnetic fields. Mathematical Methods in the Applied Sciences, 2021, 44 (16), pp.12088-12100. 10.1002/mma.6739 . hal-02975890

\section{HAL Id: hal-02975890 https://hal.science/hal-02975890}

Submitted on 25 Dec 2020

HAL is a multi-disciplinary open access archive for the deposit and dissemination of scientific research documents, whether they are published or not. The documents may come from teaching and research institutions in France or abroad, or from public or private research centers.
L'archive ouverte pluridisciplinaire HAL, est destinée au dépôt et à la diffusion de documents scientifiques de niveau recherche, publiés ou non, émanant des établissements d'enseignement et de recherche français ou étrangers, des laboratoires publics ou privés. 


\title{
Unified mathematical model of the kinetics of nanoparticle phase condensation
}

\section{in magnetic fields}

\author{
Pavel Kuzhir ${ }^{1}$, Maxime Raboisson-Michel ${ }^{1,2}$, Jordy Queiros Campos ${ }^{1}$, Gregory Verger- \\ Dubois $^{2}$, Andrey Yu. Zubarev ${ }^{3,4}$ \\ ${ }^{1}$ Université Côte d'Azur, CNRS UMR 7010, Institute of Physics of Nice, Parc Valrose, 06108 Nice, France \\ ${ }^{2}$ Axlepios Biomedical - lere Avenue 5eme rue, 06510 Carros, France \\ ${ }^{3}$ Theoretical and Mathematical Physics Department, Institute of Natural Sciences and Mathematics, Ural \\ Federal University, Lenin Ave, 51, Ekaterinburg, 620083, Russia,
}

${ }^{4}$ M.N. Mikheev Institute of Metal Physics of the Ural Branch of the Russian Academy of Sciences, Ekaterinburg, Russia

\begin{abstract}
In this paper, we aim to present a unified mathematical modelling and description of the kinetics of magnetic nanoparticles phase condensation (conducting to the appearance of bulk elongated aggregates) under homogeneous permanent or alternating magnetic field. For such case, the aggregate growth rate usually takes the form $d V / d t=G(V) \Delta(t)$, with $V$ and $t$ being the aggregate's volume and time, respectively, $\Delta(t)$ - the supersaturation of the nanoparticle suspension, and with the function $G(V)$ depending on the precise configuration of the applied field. The Liouville equation for the aggregate size distribution function is solved by the method of characteristics. The solution is obtained in parametric form for an arbitrary function $G(V)$, providing a general framework for any type of the applied magnetic field. In the particular case of low-frequency rotating magnetic field $\left(G(V) \sim V^{2 / 3}\right)$, an explicit expression of the distribution function is obtained., while the dimensionless average aggregate volume $\langle V\rangle$ is found by the method of moments allowing a complete decoupling of the system of equations for the statistical moments $\left\langle V^{n}\right\rangle$ of the distribution function. Numerical examples are provided for the cases of permanent and low- or medium frequency rotating fields. It is shown that in all cases, the average volume $\langle V\rangle$ only slightly depends on the relative width of the initial size distribution. Nevertheless, at any times, $t>0$, the size distribution shows a significant spreading around the average value $\langle V\rangle$ which increases progressively with time and achieves a final plateau at long times. This model can be helpful for several biomedical or environmental applications of magnetic nanoparticles, in which the nanoparticle suspension undergoes a field-induced phase condensation.
\end{abstract}

\section{Keywords}

Magnetic nanoparticles; non-equilibrium phase transition; magnetic field.

\section{Correspondence:}

Andrey Yu. Zubarev, ${ }^{3}$ Theoretical and Mathematical Physics Department, Institute of Natural Sciences and Mathematics, Ural Federal University, Lenin Ave, 51, Ekaterinburg, 620083, Russia.

E-mail: $\underline{\text { A.J.Zubarev@urfu.ru }}$ 


\section{Introduction}

Magnetic nanoparticles are gaining a growing interest as a versatile nanomaterial for biomedical and environmental applications $[1,2]$. In many of these applications, magnetic nanoparticles are subjected to aggregation induced by the applied external magnetic field due to dipole-dipole interactions. Such aggregation is often treated as a condensation phase transition or phase separation where initially homogeneous colloid separates into a concentrated phase (usually present in form of needle-like aggregates) and a dilute phase representing a homogeneous suspending medium for aggregates [3]. The typical aggregation timescale has often to be compared to the characteristic timescales of the given application. Kinetics of field-induced aggregation becomes therefore very important for a successful realization of the application. Since various magnetic field configurations are used in different applications (permanent, oscillatory, rotating magnetic fields), it is very useful to find a unified description of the kinetics of nanoparticle aggregation independent of the field configuration and applicable to both permanent and alternating magnetic fields. To the best of our knowledge, such generalized theory of kinetics of field-induced aggregation (or phase condensation) does not exist. Theoretical [4-6] and computer [7-9] models of the field-induced phase condensation and its kinetics have been mainly developed for the permanent magnetic field. However, the kinetics of aggregation has never been treated for the practically important case of rotating magnetic field, which makes the aggregates rotate [10, $11]$; this is expected to change dramatically the law of the aggregate growth.

In particular, in a recently proposed novel treatment of brain strokes and thrombosis, the magnetic nanoparticles are injected to the blocked vessel to activate recirculation of the blood flow near the blood clots. Its goal is to enhance the drug transport to the clot allowing its faster dissolution [12]. This recirculation is achieved by the application of rotating magnetic fields, which first induce aggregation of nanoparticles to micron-sized needle-like aggregates. Then the rotational motion of these aggregates induces recirculation flows in the blood vessel. When the applied field is switched off, the nanoparticle aggregates are destroyed by thermal motion and convective fluxes, and biocompatible nanoparticles are slowly eliminated from the human body by liver macrophages [13]. The same mechanism is involved in the elimination of magnetic nanoparticles applied as contrast agents in magnetic resonance imaging [14]. The timescale of field-induced aggregation should be much smaller than the typical time of the medical intervention. In this context, the kinetics of nanoparticle aggregation under rotating magnetic fields plays a decisive role in the success of brain stroke treatment.

This paper is focused on the development of the unified mathematical modelling of the kinetics of field-induced phase condensation in ferrfoluids under a spatially homogeneous permanent or alternating magnetic field of an arbitrary configuration (harmonic or non-harmonic oscillatory, rotating with circular or non-circular polarization, etc.). This description is given under two following limits: (a) very fast initial nucleation stage allowing the appearance of all the nuclei at very small times; (b) the aggregation timescale is large enough with respect to the characteristic period of the magnetic field. Under these limits, the unified modelling of the kinetics becomes possible since, in most of the relevant cases, the aggregate growth rate $d V / d t$ can be presented as a product of some function $G(V)$ of the aggregate volume by another function $\Delta(t)$ of time. The precise details of the aggregation mechanism are contained in the function $G(V)$, which depends on the magnetic field configuration, while the second function $\Delta(t)$ stands for the suspension supersaturation. For definiteness, two distinct cases of the permanent (with $G(V) \propto V^{3 / 7}$ ) and rotating (with $G(V) \propto V^{2 / 3}$ ) magnetic fields will be considered. The distribution function of the aggregate volume, as well as evolution of the average volume with time, will be calculated for these two particular cases as for a general case of an arbitrary function $G(V)$. 


\section{Liouville equation solution for arbitrary function $G(V)$}

In this section, we propose a mathematical model of the evolution of the dense aggregates, consisting of many magnetic nanoparticles, under a homogeneous either permanent or homogeneous alternating magnetic field. The intensity of these magnetic fields may be an arbitrary function of time, under the restriction that the period of the lowest harmonics of the magnetic field is much smaller than the aggregation timescale. In many experiments, straight elongated aggregates with a shape assimilated to prolate ellipsoids have been reported under permanent [3], rotating [10] or oscillating [3] magnetic fields, at least in a low-frequency range below a critical frequency at which the straight elongated shape becomes unstable [16]. Thus, we consider dense elongated aggregates that, depending on the magnetic field configuration, can either be stationary or perform an angular motion (rotation as an example) under the action of this field. The aggregates are illustrated in Fig.1.

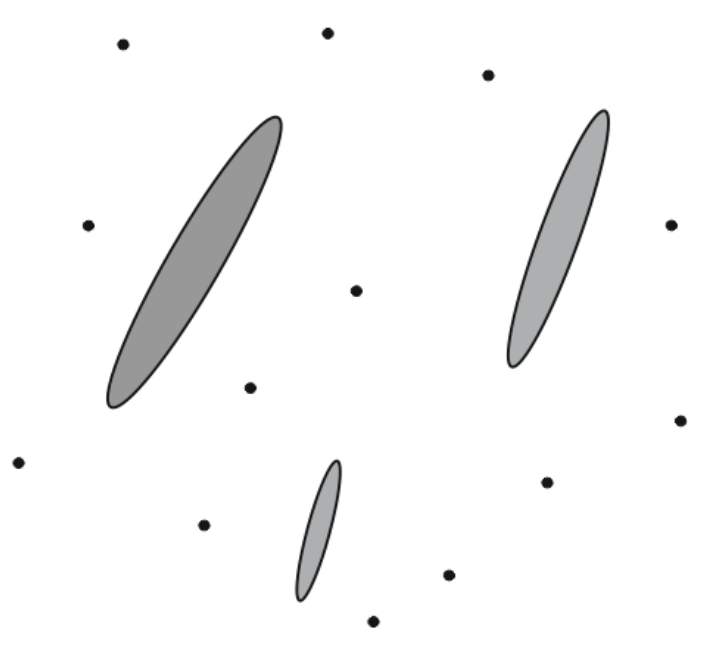

Fig.1. Sketch of the aggregates, which can either be stationary or perform an angular motion depending on the applied magnetic field. Dots around the aggregates stand for individual magnetic particles.

All real magnetic colloids (ferrofluids) are polydisperse systems, very often with a wide distribution over particles sizes. As a rule they include small particles, not able to aggregate under the magnetic forces, as well as relatively large particles with the energy of magnetic interaction large enough to provoke their aggregation in external magnetic field. Here we suppose that the aggregates appear and grow due to absorbtion of the large particles.

To prevent irreversible coagulation of the particles under the colloidal attractive forces, they are covered with special surfactant or ionic layers. However, these layers do not always completely screen the colloidal attractive forces and the particles, at the stage of the ferrofluid synthesis, form some so-called primary agglomerates (sometimes called flocculi), consisting of several or several tens of nanoparticles. That is why, the onset of the ferrofluid aggregation is determined by many factors - by the size distribution of the particles, the presence of the primary agglomerates, etc. The biggest particles or the biggest primary agglomerates can serve as centres of the heterogeneous nucleation of the main part of the particles. The rate of the primary nuclei appearance can be very high; the characteristic time of the nucleation is usually below the ability of detection of the process [17]. Therefore, the most probable scenario of the kinetics of the phase separation is the following. Once the external magnetic field is applied, for practically negligible time, the nuclei of the dense phase appear on some condensation centres. After that, due to the 
magnetic interparticle interaction, the nuclei absorb the free particles from the main part of the ferrofluid and grow; supersaturation of the system decreases. These growing nuclei will be hereinafter called aggregates. At the final stage of the phase condensation, the aggregates amalgamate because of magnetic interaction between them.

In this work, we will consider the main second part of the phase condensation, when the aggregates grow due to capture of free nanoparticles from the surrounding dilute phase. New nuclei no longer appear during this stage, while the coalescence of aggregates under attractive dipolar interaction is still negligible [17], such that the number of aggregates $N$ per unit volume will be considered to be constant with time. Because of the size polydispersity of the nucleation centres, the aggregates are expected to have unequal size. It is convenient to describe the aggregate size distribution at a given moment of time $t$ by a distribution function (probability density) $F(V, t)$ over the aggregate volumes $V$. After the very fast nucleation stage, the aggregate size usually achieve a few microns. The effect of thermal fluctuations on the rate of volume growth is expected to be negligible for such supercritical aggregates. That is why, as commonly admitted in the theory of phase transitions, the distribution function $F(V, t)$ of the aggregate size obeys the classical Liouville equation free of any diffusive terms [18]:

$\frac{\partial F}{\partial t}+\frac{\partial}{\partial V}(\dot{V} F)=0$

with the normalization condition

$\int_{0}^{\infty} F(V, t) d V=1$

and the initial condition

$F(V, 0)=F_{0}(V)$

relevant for the considered case of constant number density $N$ of aggregates with the initial size distribution $F_{0}(V)$ in the issue of very short heterogeneous nucleation stage. In the present work, this initial distribution is supposed to be known, while in practice it can either be measured in experiments or modelled through a detailed consideration of the heterogeneous nucleation stage.

The aggregate growth rate $d V / d t$ depends on both the aggregate volume $V$ and time $t$. However, in most physically relevant cases, separation of variables takes place and the function $d V / d t$ takes the following general form:

$\dot{V}(V, t)=G(V) \Delta(t), \quad \dot{V} \equiv \frac{d V}{d t}$

where $\Delta(t)=\varphi(t)-\varphi_{0}$ is the ferrofluid supersaturation, $\varphi(t)$ is the mean volume concentration of the free particles in the inter-aggregate space (in the dilute phase) and $\varphi_{0}$ is the concentration of the nanoparticles in the dilute phase when the equilibrium between the dilute and concentrated (inside the aggregate) phases is achieved. The function $G(V)$ depends on the intensity, frequency and shape of the temporal dependency of the magnetic field. For example, in the case of the permanent magnetic field, $G(V) \propto V^{3 / 7}$ [19], while in the case of the circularly polarized rotating field, $G(V) \propto V^{2 / 3}$ at low frequency and can show an intermediate behaviour between $V^{3 / 7}$ and $V^{2 / 3}$ at higher frequencies, as shown in the Appendix.

The size distribution must also respect the volume conservation condition that can be written in terms of the supersaturation as follows [17, 19]:

$\Delta(t)=\Delta_{0}-N \varphi_{i}\langle V\rangle$,

$\langle V\rangle=\int_{0}^{\infty} F(V, t) V d V$, 
where $\varphi_{i}$ is the volume fraction of nanoparticles inside the aggregates and $\langle V\rangle$ is the average aggregate volume at a given time $t$.

Equation (5a) allows us to determine the maximal average volume of the aggregates at the end of the aggregate growth stage when the supersaturation tends to zero: $\langle V\rangle_{\max }=\Delta_{0} /\left(N \varphi_{i}\right)$. It is thus reasonable to scale the aggregate volume by the maximal average volume and introduce the dimensionless aggregate volume as $v=V /\langle V\rangle_{\max }$. Furthermore, the function $G(V)$ has a dimension of the aggregate growth rate $\left(\mathrm{m}^{3} / \mathrm{s}\right)$ and can be presented as

$G(V) \equiv \kappa\langle V\rangle_{\max } g(v)$,

with $\kappa$ being some kinetic constant having a dimension of inverse time $\left(\mathrm{s}^{-1}\right)$, and $g(v)-$ a function of the dimensionless volume $v$ that can in principle depend on $\langle V\rangle_{\max }$. With this in mind, let us introduce the following dimensionless quantities: $f=\langle V\rangle_{\max } F-$ the dimensionless distribution function; $\tau=\Delta_{0} \kappa t-$ the dimensionless time and $\omega=\Delta / \Delta_{0}-$ the reduced supersaturation. The initial value problem (1) - (5) for the aggregate size distribution can now be written in the following dimensionless form:

$\frac{\partial f}{\partial \tau}+\omega(\tau) \frac{\partial}{\partial v}(g(v) f)=0$,

$\int_{0}^{\infty} f(v, \tau) d v=1$,

$f(v, 0)=f_{0}(v)$,

$\omega(\tau)=1-\langle v\rangle=1-\int_{0}^{\infty} f(v, \tau) v d v$

Introducing new functions:

$U(v, \tau)=g(v) f(v, \tau)$,

$\theta(\tau)=\int_{0}^{\tau} \omega\left(\tau^{\prime}\right) d \tau^{\prime}$

$y(v)=\int_{0}^{v} \frac{d v^{\prime}}{g(v \prime)}$

we come to the problem with the initial condition as follows:

$\frac{\partial U}{\partial \Theta}+\frac{\partial U}{\partial y}=0, \quad U(y, 0)=g(v(y)) f_{0}(v(y))$,

where $v(y)$ is the inverse function of $y(v)$ defined in $(7 \mathrm{c})$.

By using the methods of characteristics, one can get the solution of the initial value problem (8) as

$U(y, \theta)=g(v(y-\theta)) f_{0}(v(y-\theta)) H(y-\theta)$,

where $H(x)$ is the Heaviside step function, introduced to avoid negative values of the argument (dimensionless volume) of the initial size distribution $f_{0}(v)$. Let us denote $v(y-\theta)=v^{*}(y, \theta)$. Since $v(y)$ is the inverse function of $y(v)$ than $v(y-\theta)$ is expected to be an inverse function of $y(V)-\theta(\tau)$. This implies $y\left(v^{*}\right)=y(v)-\theta(\tau)$. Using the definition of $y$ [Eq. (7c)], we get:

$\theta=\int_{v^{*}}^{v} \frac{d v^{\prime}}{g\left(v^{\prime}\right)} \rightarrow v^{*}=$ function $(\theta, v)$.

With this new notation, the distribution function takes the following form in coordinates $(v, \theta)$ :

$f(v, \theta)=\frac{1}{g(v)} U(v, \theta)=\frac{1}{g(v)} g\left(v^{*}(v, \theta)\right) f_{0}\left(v^{*}(v, \theta)\right) H\left(v^{*}(v, \theta)\right)$, 
where $v^{*}(v, \theta)$ is the solution of Eq. (10). From the solution (11), it follows that at a given equivalent time $\theta$, the dimensionless volume $v$ has a minimal non-zero value $v_{\text {min }}$ defined by the condition $v^{*}\left(v_{\text {min }}, \theta\right)=0$. Substituting this expression to Eq. (10), we get the following transcendental equation allowing finding the minimal volume as a function of $\theta$ :

$\theta=\int_{0}^{v_{\min }} \frac{d v^{\prime}}{g\left(v^{\prime}\right)} \rightarrow v_{\min }=$ function $(\theta)$.

Before finding $\theta(\tau)$, let us first find the average aggregate volume as a function of the unknown $\theta$.

$\langle v\rangle=\int_{0}^{\infty} f(v, \theta) v d v=\int_{v_{\min }(\theta)}^{\infty} \frac{g\left(v^{*}(v, \theta)\right) f_{0}\left(v^{*}(v, \theta)\right)}{g(v)} v d v=$ function $(\theta)$.

Then we use the volume conservation condition (6d), $\omega(\tau)=d \theta / d \tau=1-\langle v\rangle$, whose solution is trivial:

$\tau(\theta)=\int_{0}^{\theta} \frac{d \theta^{\prime}}{1-\langle v\rangle\left(\theta^{\prime}\right)}$.

Thus, the average volume and the distribution function can be found in the function of time in parametric form by variation of the parameter $\theta$. The main steps of such parametric calculation are summarized as follows:

1) first, we find the minimal volume $v_{\min }(\theta)$ by solving the transcendental equation (12);

2) then, we find $v^{*}\left(v_{\min }, \theta\right)$ by solving the transcendental equation (10);

$3)$ then we find, the average volume $\langle v\rangle(\theta)$ and time $\tau(\theta)$ by Eqs. (13), (14);

4) finally, we plot the distribution function $f(v, \theta)$ as a function of $v$ at different moments of time $\tau(\theta)$.

This procedure can be applied to an arbitrary function $g(v)$ depending on the magnetic field configuration. In the next two sections, we focus our attention on a specific form of the function $g(v)=v^{2 / 3}$, relevant for rotating magnetic field and allowing more concise analytical solution for $f(v, \theta)$ and $\tau(\theta)$ (Sec. III) and finding the average volume $\langle v\rangle$ by the method of moments without solving the Liouville equation (Sec. IV).

\section{Resolution of the Liouville equation for the particular case $g(v)=v^{2 / 3}$}

The particular case $g(v)=v^{2 / 3}$ corresponds to the circularly polarized rotating magnetic field (see Appendix) and is important for the application of magnetic nanoparticles in magnetically assisted brain stroke treatment, as pointed out in Introduction. This case allows rather simple expressions for all unknown quantities, such as $v_{\text {min }}, v^{*}, \theta$ and $f(v, \theta)$. Straightforward application of the calculation steps described at the end of Sec. II gives the following results:

$$
\begin{aligned}
& v_{\min }=\left(\frac{\theta}{3}\right)^{3} \\
& v^{*}=\left(v^{1 / 3}-\frac{\theta}{3}\right)^{3} \\
& \langle v\rangle=\int_{v_{\min }(\theta)}^{\infty} v^{* 2 / 3} f_{0}\left(v^{*}\right) v^{1 / 3} d v=3 \int_{0}^{\infty} \xi^{2} f_{0}\left(\xi^{3}\right)\left(\xi+\frac{\theta}{3}\right)^{3} d \xi=A+B \theta+C \theta^{2}+D \theta^{3} \\
& \tau(\theta)=\int_{0}^{\theta} \frac{d \theta^{\prime}}{1-\left[A+B \theta^{\prime}+C \theta^{\prime 2}+D \theta^{\prime 3}\right]} \\
& f(v, \theta)=v^{-2 / 3}\left(v^{1 / 3}-\frac{\theta}{3}\right)^{2} f_{0}\left(\left(v^{1 / 3}-\frac{\theta}{3}\right)^{3}\right) H\left(v^{1 / 3}-\frac{\theta}{3}\right)
\end{aligned}
$$


$A=3 \int_{0}^{\infty} \xi^{5} f_{0}\left(\xi^{3}\right) d \xi ; B=3 \int_{0}^{\infty} \xi^{4} f_{0}\left(\xi^{3}\right) d \xi ; C=\int_{0}^{\infty} \xi^{3} f_{0}\left(\xi^{3}\right) d \xi ; D=\frac{1}{9} \int_{0}^{\infty} \xi^{2} f_{0}\left(\xi^{3}\right) d \xi$

where we have made a change of variables $\xi \equiv v^{* 1 / 3}=v^{1 / 3}-\theta / 3$ when calculating the integral in $(15 \mathrm{c})$. The integral in (15d) can be evaluated numerically, while the numerical values of the coefficients $A, B, C$ and $D$ in (15f) will depend on the shape of the initial size distribution $f_{0}(v)$. Numerical examples will be analyzed in Sec. V.

The analysis shows that in the particular case of the Dirac delta initial distribution of the aggregate size $f_{0}(v)=\delta\left(v-v_{0}\right)$, i.e. all aggregates of the same initial volume $v_{0}$, the size distribution remains monodisperse at any moment of time $\tau$ or $\theta$ :

$f(v, \theta)=\delta\left(v-v_{1}\right)$

with the aggregate volume $v_{1}$ (the same for all aggregates) and the dimensionless time $\tau$ given by

$v_{1}=\left(v_{0}^{1 / 3}+\frac{\theta}{3}\right)^{3} ; \tau(\theta)=\int_{0}^{\theta} \frac{d \theta^{\prime}}{1-\left(v_{0}^{1 / 3}+\theta^{\prime} / 3\right)^{3}}$,

or, eliminating $\theta$ from (17), we get the following explicit expression for the dimensionless time as function of the aggregate volume in this particular case of monodisperse size distribution:

$\tau\left(v_{1}\right)=\int_{v_{0}}^{v_{1}} \frac{d v}{v^{\frac{2}{3}}(1-v)}=\psi\left(v_{1}\right)-\psi\left(v_{0}\right) ; \quad \psi(x)=3^{1 / 2} \operatorname{atan} \frac{1+2 x^{1 / 3}}{3^{1 / 2}}+\frac{1}{2} \ln \frac{1+x^{1 / 3}+x^{2 / 3}}{\left(1-x^{1 / 3}\right)^{2}}$

The case of monodisperse aggregate size will be compared to the case of the polydisperse size distribution (with some initial distribution $f_{0}(v) \neq \delta\left(v-v_{0}\right)$ ) in Sec. V.

\section{Method of moments for determination of the average volume in particular case $g(v)=v^{2 / 3}$.}

In this section, we show that the special case $g(v)=v^{2 / 3}$ admits a complete decoupling of the moments $\left\langle v^{n}\right\rangle=\int_{0}^{\infty} v^{n} f(v) d v, n=\frac{1}{3}, \frac{2}{3}, 1$ of the distribution function $f(v, \tau)$ without any closure approximation. Thus, we do not need to solve the Liouville equation (6a) for the distribution function to find the average volume $\langle v\rangle$. Such decoupling of moments is of particular interest for statistical physics and can generally provide faster computations as compared to the general method provided in Sec. III.

First, in the considered case, $g(v)=v^{2 / 3}$, the Liouville equation (6a) written in dimensionless variables takes the following form:

$\frac{\partial f}{\partial \tau}+\omega(\tau) \frac{\partial}{\partial v}\left(v^{2 / 3} f\right)=0$

Then, multiplying both parts of Eq. (19) by $v$ and integrating over $v$, one gets:

$\frac{d<v>}{d \tau}+\omega \int_{0}^{\infty} v \frac{\partial}{\partial v}\left(v^{2 / 3} f\right) d v=0$.

Integrating by parts, taking into account that $f=0$ at $v=0, v \rightarrow \infty$, we come to the equation:

$\frac{d\langle v\rangle}{d \tau}=\omega<v^{2 / 3}>$

Similarly, multiplying Eq. (19) by $v^{2 / 3}$ and by $v^{1 / 3}$, and integrating over $v$, we get respectively: 
$\frac{d\left\langle v^{2 / 3}\right\rangle}{d \tau}=\frac{2}{3} \omega<v^{1 / 3}>$

$\frac{d<v^{1 / 3}>}{d \tau}=\frac{1}{3} \omega$

Along with the volume conservation equation (6d), implying $\omega(\tau)=1-\langle v\rangle$, equations (20) - (22) present a closed system of differential equations with respect to the moments $\langle v\rangle$ $,\left\langle v^{2 / 3}\right\rangle,\left\langle v^{1 / 3}\right\rangle$. Eliminating the moments $\left\langle v^{2 / 3}\right\rangle$ and $\langle v\rangle$, we arrive at a single differential equation as follows for the moment $\left\langle v^{1 / 3}\right\rangle$ :

$\frac{d<v^{1 / 3}>}{d \tau}=\frac{1}{3}\left[1-\left(<v^{1 / 3}>^{3}+3 C_{1}<v^{1 / 3}>+C_{2}\right)\right]$

where the integration constants $C_{1}$ and $C_{2}$ are found from the initial size distribution $f_{0}(v)$ as follows:

$C_{1}=\left\langle v^{2 / 3}\right\rangle_{0}-\left(\left\langle v^{1 / 3}\right\rangle_{0}\right)^{2}, C_{2}=\langle v\rangle_{0}+2\left(\left\langle v^{1 / 3}\right\rangle_{0}\right)^{3}-3\left\langle v^{2 / 3}\right\rangle_{0}\left\langle v^{1 / 3}\right\rangle_{0}$

with $\left\langle v^{n}\right\rangle_{0}=\int_{0}^{\infty} v^{n} f_{0}(v) d v, n=\frac{1}{3}, \frac{2}{3}, 1$.

The differential equation (23) allows expressing the moment $\left\langle v^{1 / 3}\right\rangle$ as an inverse function of the dimensionless time $\tau$, while the system (20)-(22) allows one to relate the average volume $\langle v\rangle$ to $\left\langle v^{1 / 3}\right\rangle$. Thus, the time dependence of the average volume can be written in the following parametric form using $\left\langle v^{1 / 3}>\equiv x\right.$ as a parameter:

$<v>(x)=x^{3}+3 C_{1} x+C_{2}$,

$\tau(x)=3 \int_{x_{0}}^{x} \frac{d x^{\prime}}{1-\left(x^{3}+3 C_{1} x^{\prime}+C_{2}\right)}, x_{0}=\left\langle v^{1 / 3}\right\rangle_{0}$.

It can be easily checked, that in particular case of monodisperse initial size distribution, $f_{0}(v)=\delta\left(v-v_{0}\right)$, the constants $C_{1}=C_{2}=0$, the size distribution remains monodisperse with time and given by Eq. (16), while the aggregate volume $v_{1}$ (the same for all aggregates) is given by the inverse function of time provided in Eq. (18).

\section{Results}

For the sake of the definiteness, we apply our models developed in Secs. II-IV to the physically relevant case of initial log-normal distribution of aggregate sizes:

$f_{0}(v)=\frac{1}{\sqrt{2 \pi} \sigma v} \exp \left(-\frac{\left(\ln \left(\frac{v}{\langle v\rangle_{0}}\right)+\frac{\sigma^{2}}{2}\right)^{2}}{2 \sigma^{2}}\right) H(v)$,

where $\langle v\rangle_{0}$ is the initial dimensionless average aggregate volume and $\sigma$ is a parameter characterizing the distribution width.

Let us first analyze the evolution of the dimensionless average aggregate volume $\langle v\rangle$ with dimensionless time $\tau$ for the three following cases:

(a) a permanent magnetic field with $g(v)=v^{3 / 7}$ (as shown in $[17,19]$ ) - the case labelled as "P";

(b) low frequency circularly polarized rotating magnetic field with $g(v)=v^{2 / 3}$ (see Appendix) the case labelled as "R1";

(c) moderate frequency circularly polarized magnetic field with 


$$
g(v)=\left[a v^{-2 / 3}+b v^{-3 / 7}\right]^{-1}
$$

where $a$ and $b$-dimensionless constants (see Appendix) - the case labelled as "R2".

All cases are treated using the general methodology developed at the end of Sec. II, while it is checked that for the case $\mathrm{R} 1\left(g(v)=v^{2 / 3}\right)$, the calculations using this methodology (applied in Sec. III for this case) and the method of moments (developed in Sec. IV) coincide. The $\langle v\rangle$ versus $\tau$ dependency is plotted in Fig. 2 for the three cases with the initial log-normal size distribution [Eq. (26)] characterized by $\langle v\rangle_{0}=0.1$ and $\sigma=1.5$. The case R2 is calculated at $a=b=1$.

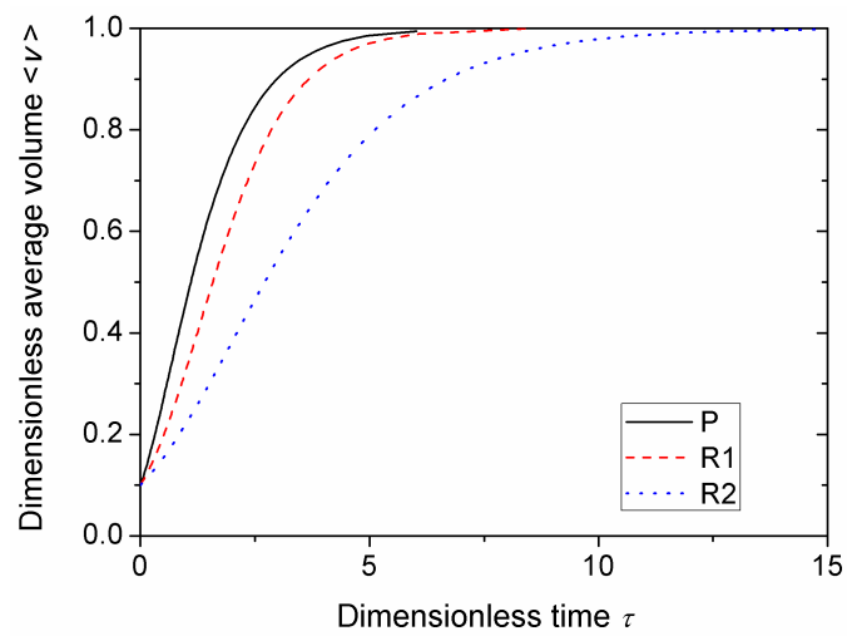

Fig. 2. Dimensionless average aggregate volume $\langle v\rangle$ as function of dimensionless time $\tau$ for three different functions $g(v)$ and initial log-normal size distribution with $\langle v\rangle_{0}=0.1$ and $\sigma=1.5$. The case $\mathrm{R} 2$ is calculated for $a=b=1$.

As is seen from this figure, the average aggregate volume gradually increases with time from its initial value $\langle v\rangle_{0}=0.1$ and asymptotically tends to unity at long times. Recalling that the dimensional volume $V$ has been normalized by the maximal average volume, $\langle V\rangle_{\max }=$ $\Delta_{0} /\left(N \varphi_{i}\right)$, this means that at long times the average dimensional volume $V$ tends to its maximal value when the ferrofluid supersaturation $\Delta$ (or $\omega$ ) tends to zero. We have to bear in mind that the coalescence of aggregates has been neglected at this stage, and in fact, our preliminary experiments show that coalescence is strongly hindered by hydrodynamic repulsion between aggregates in the case of rotating magnetic field, which makes the aggregate turn synchronously with the field. Analyzing the difference between the three plotted curves in Fig. 2, one could presume that the permanent magnetic field (curve "P") provides a faster aggregation than the rotating one, while low-frequency rotating field (curve "R1") ensures faster aggregation than medium-frequency rotating field (curve "R2"). We have to bear in mind however that the kinetic constant $\kappa$ (introduced below Eq. (5)) can be essentially different for three considered cases implying different dimensional times $t=\tau /\left(\Delta_{0} \kappa\right)$ at the same dimensionless time $\tau$. For example, in a permanent magnetic field, the kinetic constant is related to the inverse characteristic time of Brownian diffusion of a single nanoparticle. On the other hand, in a rotating field, the kinetic constant is expected to be a function of the field frequency while the convective transport of nanoparticles towards the aggregate is expected to be enhanced with increasing frequency leading to faster aggregation at a higher frequency. At this stage, we restrict our analysis to the dimensionless quantities and focus our attention to a general theoretical description of the solution of the kinetic problem. A deeper insight into the physical aspects related to precise dependence of the kinetic constant on the dimensional physical parameters (as magnetic field intensity, frequency, particle size, etc.) will be provided in future in comparison with experiments. 
Let us now inspect the effect of the width $\sigma$ of the initial size distribution [Eq. (26)] on the average volume. The $\langle v\rangle$ versus $\tau$ dependency is plotted in Fig. 3 for the case R1 $\left(g(v)=v^{2 / 3}\right)$ for $\langle v\rangle_{0}=0.1$ and three different values of $\sigma$, with $\sigma=0$ corresponding to the Dirac delta initial distribution admitting the analytical solution Eq. (18).

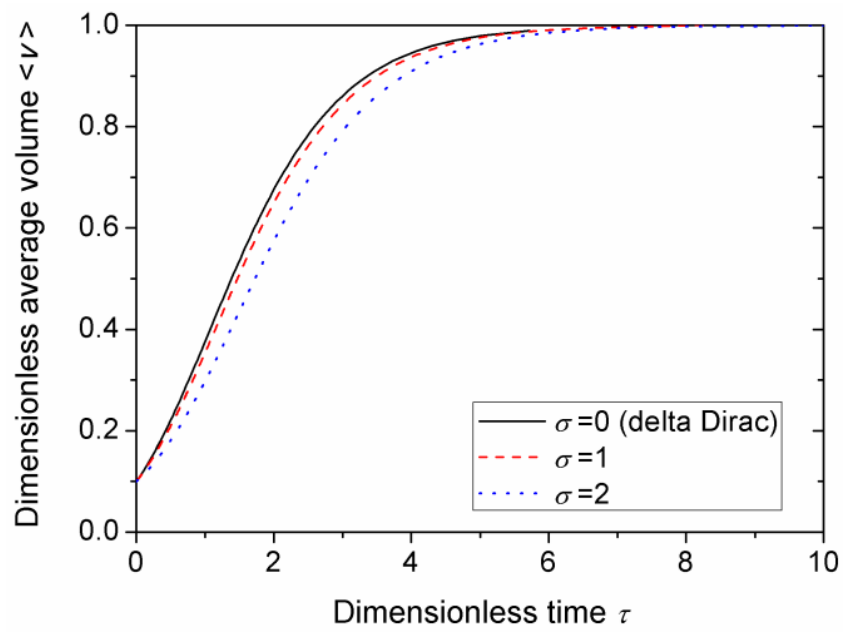

Fig. 3. Dimensionless average aggregate volume $\langle v\rangle$ as function of dimensionless time $\tau$ for the R1-case and initial log-normal size distribution with $\langle v\rangle_{0}=0.1$ and three different values $\sigma$ of the distribution width.

As is seen from this figure, the curves are shifted to the right with increasing the initial distribution width ( $\sigma$ parameter), indicating that the aggregation gets slower. However, the difference with the monodisperse distribution (Dirac delta with $\sigma=0$ ) remains really small even at a very wide initial distribution with $\sigma=2$. Such a difference can be on the order of errors related to different model assumptions and it seems reasonable to use simple analytical result [Eq. (18)] (derived for monodisperse distribution) for the average aggregate volume even in the case of wide initial size distribution.

Having found a slight effect of the initial distribution width, it is now interesting to inspect what happens with the size distribution with time. The distribution function $f(v)$ of the dimensionless aggregate volume is plotted in Fig. 4 for the R1-case with $\langle v\rangle_{0}=0.1, \sigma=1.5$ and three different dimensionless times, including the initial moment $\tau=0$ with a given initial distribution $f_{0}(v)$ [Eq. (26)] and the moment of time $\tau=8.4$ at which the average aggregate volume is equal to $\langle v\rangle=0.999$ (thus approaches its maximal value $\langle v\rangle_{\max }=1$ ).

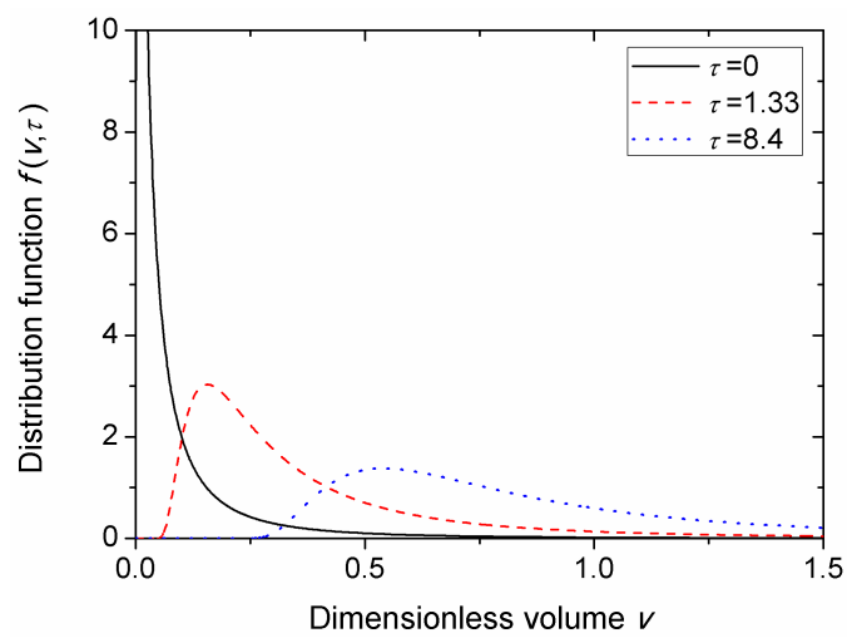

Fig. 4. Aggregate size distribution for the R1-case with $\langle v\rangle_{0}=0.1, \sigma=1.5$ and at the dimensionless times $\tau$, covering the whole range of the average volume $\langle v\rangle_{0} \leq\langle v\rangle \lesssim 1$. 
It is seen from Fig. 4 that the size distribution displaces along the abscissa axis and spreads over this axis during time although the average volume remains close to the one of the monodisperse distribution. One can, therefore, conclude that the distribution globally keeps its initial shape and nearly equally spreads around its average value roughly given by the monodisperse approximation [Eq. (18)].

For the practical biomedical applications, the whole size distribution (and not only the average aggregate volume) could appear to be an important parameter. For example in brain stroke treatments, the largest aggregates can achieve the size of the vessels and the smallest aggregates can be too small for effective manipulation by external fields. It was, therefore, important to learn about relatively large width of the aggregate size distribution (Fig. 4) even in the case when the average aggregate volume was nearly independent of the width of initial distribution (Fig. 3).

\section{Concluding remarks}

In this paper, we present a mathematical model of nanoparticle phase condensation (or, equivalently, nanoparticle aggregation) under homogeneous permanent or homogeneous alternating magnetic field with arbitrary time dependence of the magnetic field intensity. The model is derived under basic assumptions of very fast nucleation kinetics but negligible aggregate coalescence (thus, the aggregate number density is constant over time) and for the magnetic field frequency range respecting two following conditions: (a) the aggregate growth is slow enough with respect to the field variation, and (b) the aggregates keep their straight elongated shape. Under these conditions, the aggregate growth rate takes the general form $d V / d t=G(V) \Delta(t)$, with the function $G(V)$ depending on the precise configuration of the applied magnetic field. The ensemble of aggregates is described by their size distribution function $F(V, t)$, which is found by the solution of the Liouville equation (1) at an appropriate initial condition. The problem is solved by the method of characteristics, and the solution is obtained in parametric form for an arbitrary function $G(V)$, providing a general framework for any type of the applied magnetic field. In its dimensionless form, the solution is expressed through the integrals, whose integrands contain the dimensionless counterparts, $g(v)$ and $f_{0}(v)$, of the function $G(V)$ and the initial size distribution $F_{0}(V)$, respectively. In the particular case of low-frequency rotating magnetic field $\left(g(v)=v^{2 / 3}\right)$, an explicit expression of the dimensionless distribution function $f(v, \theta)$ is obtained [Eq. (15e)], while the dimensionless average aggregate volume $\langle v\rangle$ is easily found by the method of moments allowing a complete decoupling of the statistical moments $\left\langle v^{n}\right\rangle, n=\frac{1}{3}, \frac{2}{3}, 1$, without the necessity to solve the Liouville equation.

Numerical examples are provided for initial log-normal size distribution and the cases of a permanent magnetic field $\left(g(v)=v^{3 / 7}\right)$, low-frequency rotating field $\left(g(v)=v^{2 / 3}\right)$, and medium-frequency rotating field (with intermediate behaviour between $v^{3 / 7}$ and $v^{2 / 3}$ ). All these cases share the following common features: (a) at long times, the average volume asymptotically tends to its maximal value $\langle V\rangle_{\max }=\Delta_{0} /\left(N \varphi_{i}\right)$ (or, $\langle v\rangle_{\max }=1$ in the dimensionless form) when the ferrofluid supersaturation $\Delta$ (or $\omega$ ) tends to zero; (b) the average volume depends only slightly on the relative width $\sigma$ of the initial size distribution, allowing one to evaluate $\langle v\rangle$ for any $\sigma$ (within the range $0 \leq \sigma \leq 2$ ) using the explicit expression [Eq. (18)] derived for the Dirac delta distribution; (c) despite a negligible dependence of the average volume on $\sigma$, the width of the size distribution at any moment $\tau>0$ strongly depends on $\sigma$; the size distribution shifts along the abscissa axis with time and shows a significant spreading around the average value $\langle v\rangle$, however, this spreading seems to be quasi-symmetric with respect to $\langle v\rangle$, such that the average volume appears to be slightly affected by the width of the initial distribution. The three considered cases 
show some difference when the average dimensionless volume $\langle v\rangle$ is plotted against the dimensionless time $\tau$, however, at the tendency can change if the analysis is conducted in dimensional quantities because the kinetic constant $\kappa$ (which intervenes into the normalization of the dimensional time $t$ ) depends on the physics behind each particular case (permanent or rotating magnetic field). A deeper insight into this problem will be presented in future in conjunction with the comparison with experiments.

From the practical perspective, it was important to learn about relatively large width of the aggregate size distribution (Fig. 4) even in the case when the average aggregate volume was nearly independent of the width of initial distribution (Fig. 3). In fact, the presence of too small or too large aggregates can be undesirable in biomedical or environmental applications of magnetic nanoparticles, for example, in brain stroke treatment. Thus, the mathematical model of aggregation kinetics presented in this work can provide useful feedback to a given application allowing optimization of operating parameters.

\section{Appendix. The rate of the aggregate growth in a rotating field}

In this Appendix, we derive a scaling law of the aggregate growth rate $\dot{V}$ (the increase of its volume by unit time) as a function of the aggregate volume. At this stage, we are not looking for the exact expression for this rate, as long as it does not affect the mathematical structure of the kinetic equation (6a) considered in Sec. II and the complex physics of the aggregate growth only affect the kinetic constant $\kappa$ in the scaling law $d V / d t=$ function $(V)$. We consider a dense aggregate, of a length $L$ and diameter $d_{a}$ consisting of many ferromagnetic nanoparticles, elongated by the applied rotating field and synchronously spinning with this field. The angular frequency of the rotating field is denoted by $\Omega$. These aggregates are illustrated in Fig.1. We denote $\varphi_{i}=O(1)$ - the volume fraction of nanoparticles inside the aggregates Kinetics of the aggregate growth is described by the following approximate balance equation:

$\varphi_{i} \dot{V}=J$,

where $J$ is the flux of the particles toward the aggregate.

The magnetophoretic flux arising due to magnetic attraction of individual nanoparticles to strongly aggregates has been found to be negligible in comparison to the diffusive flux $[17,19]$, while the typical ratio of the convective to the diffusive flux, at the distances comparable with the aggregate diameter $d_{a}$, is estimated through the Péclet number: $P e=L \Omega d_{a} / D$, where $D$ is the Brownian diffusivity of a single nanoparticle. Estimates show that for individual nanoparticles of typical size $\sim 20 \mathrm{~nm}$ and the typical aggregates with a diameter $d_{a} \sim 1-10 \mu \mathrm{m}$ and length $L \sim 100 \mu \mathrm{m}$ suspended in water under the action of the field rotating with the frequency $\Omega \sim 10 \mathrm{~s}^{-1}$ , the strong inequality $P e>>1$ holds. At such condition, the surface density of the diffusive flux on the aggregate surface can be evaluated using the boundary layer approach [20]:

$j_{D} \sim D \Delta / \delta ; \quad \delta \sim d_{a} P e^{-1 / 3}$.

Here $\Delta$ is the ferrofluid supersaturation (see Sec. II) and $\delta$ is the thickness of the diffusion boundary layer near the aggregate surface. According to the concept of the boundary layer approach, outside this layer, the convective flux dominates over the diffusion one; while the diffusion flux dominates inside the layer. Integrating the flux density [Eq. (A.2)] over the aggregate surface, one gets the following scaling for the particle flux (and consequently aggregate growth rate):

$\dot{V} \sim J \sim D L P e^{1 / 3} \Delta$,

The aggregate length and diameter are related to its volume by the following scaling $[17,19]$ :

$L \propto V^{3 / 7}$ and $d_{a} \propto V^{2 / 7}$ 
Combining Eqs. (A.3), (A.4), with the definition of the Péclet number, we arrive at the following scaling relationship for the aggregate growth rate:

$\dot{V} \propto V^{\frac{2}{3}} \Delta(t)$

where a dimensional multiplier before $V^{2 / 3} \Delta$ depends on the field frequency $\Omega$ and the nanoparticle diffusivity $D$; however, derivation of the exact expression for this multiplier is out of the scope of the present work, as long as it does not affect the mathematical structure of the kinetic equation.

Notice that in reality, the relationship $d V / d t=$ function $(V)$ can appear to be more complicated. In fact, the aggregate rotates in some volume having a disk-like shape and after one spin the aggregate can absorb most of the particles situated inside this disk-like cavity. In such situation, the free nanoparticles will diffuse from outside towards the cavity and the total flux will be affected by both convective transport of particles inside the cavity towards the aggregate and purely diffusive transport of particles towards the cavity. From obvious electric analogy, one can deduce that the total flux follows the rule of the series circuit with the inverse of the equivalent conductivity (the proportionality factor between $J$ and $\Delta$ ) being the sum of inverse conductivities of purely convective [Eq. (A.5)] and purely diffusive $\left(J \sim V^{3 / 7} \Delta-\right.$ see $\left.[17,19]\right)$ transports. In such circumstances we get

$d V / d t \propto\left(\alpha V^{-\frac{2}{3}}+\beta V^{-\frac{3}{7}}\right)^{-1} \Delta(t)$,

with the dimensional constants $\alpha$ and $\beta$ depending on exact expressions for the diffusive and convective fluxes. The term in parentheses in Eq. (A.6) is nothing but the function $G(V)$, intervening into the aggregate growth rate [Eq. (4)] with its dimensionless counterpart taking the form $g(v)=\left[a v^{-2 / 3}+b v^{-3 / 7}\right]^{-1}$, where $a$ and $b$-dimensionless constants proportional to $\alpha$ and $\beta$, respectively.

Notice that equation (A.6) is expected to apply at intermediate field frequencies, which remain small enough such that straight elongated shape of the aggregate is still stable, while Eq. (A.5) is expected to apply for relatively low frequencies when, after one aggregate spin, the nanoparticles have enough time to fill the cavity and compensate the "loss" of the "cavity particles" absorbed by the aggregate. The frequency has to be however high enough such that the aggregation timescale is always much slower than the rotation period and the high Péclet number limit is still sasisfied.

\section{Acknowledgements}

PK acknowledges the French 'Agence Nationale de la Recherche', Project Future Investments UCA JEDI, No. ANR-15-IDEX-01 (projects ImmunoMag and MagFilter) and the private company Axlepios Biomedicals for financial support, JQC acknowledges the financial support of UCA JEDI and Axlepios Biomedicals through the PhD fellowship. AZ thanks the Russian Science Foundation, project 20-12-00031, for the financial support.

\section{Authors contribution.}

PK - the main idea of this work, writing and revising the manuscript, obtaining the funding; MRM - participation in the development of the general framework (Sec. II); JQC - participation in the modelling of the special case (Sec. III and IV); GVD - funding of the studies and practical 
recommendations for given biomedical applications; AZ - development of the main part of the mathematical model.

\section{Conflict of interests}

Authors declare no potential conflict of interests

\section{ORCID}

Andrey Zubarev 0000-0001-5826-9852

Pavel Kuzhir 0000-0001-7089-6197

\section{References}

[1] Tartaj, P., Morales, M. P., Veintemillas-Verdaguer, S., Gonzalez-Carreño, T., \& Serna, C. J. (2006). Synthesis, properties and biomedical applications of magnetic nanoparticles. Handbook of magnetic materials, 16(5), 403-482.

[2] Pratt, A. (2014). Environmental applications of magnetic nanoparticles. In Frontiers of Nanoscience (Vol. 6, pp. 259-307). Elsevier.

[3] Socoliuc, V., Vékás, L., \& Turcu, R. (2013). Magnetically induced phase condensation in an aqueous dispersion of magnetic nanogels. Soft Matter, 9(11), 3098-3105.

[4] Tsebers, A.O. Thermodynamic stability of magnetofluids. Magnetohydrodynamics 1982 18, 137-142.

[5] Sano, K; Doi, M. Theory of Agglomeration of Ferromagnetic Particles in Magnetic Fluids J. Phys. Soc. Jpn. 1983 $52,2810-2815$.

[6] Buyevich, Yu. A.; Ivanov A. O.. Physica A, 1992, 190, 276-294

[7] Satoh, A.; Chantrell, R.W.; Kamiyama, S.I.; Coverdale, G.N. Three Dimensional Monte Carlo Siulations of Thick Chai-like Clusters Composed of Ferromagnetic Fine Particles. Journal of Colloid and Interface Science, 1996, 181, $422-428$

[8] Hynninen, A.; Dijkstra M. Phase Diagram of Dipolar Hard and Soft Spheres: Manipulation of Colloidal Crystal Structures by an External Field; Physical Rev. Letters, 2005 94, 138303

[9] Hynninen, A.; Dijkstra M., Phase behavior of dipolar hard and soft spheres, Physical Rev.E., 2005 72, 051402

[10] Sandre, O., Browaeys, J., Perzynski, R., Bacri, J. C., Cabuil, V., \& Rosensweig, R. E. (1999). Assembly of microscopic highly magnetic droplets: Magnetic alignment versus viscous drag. Physical Review E, 59(2), 1736.

[11] Stikuts, A. P., Perzynski, R., \& Cēbers, A. (2020). Spontaneous order in ensembles of rotating magnetic droplets. Journal of Magnetism and Magnetic Materials, 500, 166304.

[12] Creighton, Francis M. 2012."Magnetic-based systems for treating occluded vessels." U.S. Patent No. 8,308,628. 13 Nov.

[13] Ali, A., Hira Zafar, M. Z., ul Haq, I., Phull, A. R., Ali, J. S., \& Hussain, A. (2016). Synthesis, characterization, applications, and challenges of iron oxide nanoparticles. Nanotechnology, science and applications, 9, 49.

[14] Ito, A.; Shinkai, M.; Honda, H.; Kobayashi, T. Medical application of functionalized magnetic nanoparticles. J. Biosci. Bioeng. 2005, 100, 1-11.

[15] Promislow, J. H., \& Gast, A. P. (1997). Low-energy suspension structure of a magnetorheological fluid. Physical Review E, 56(1), 642.

[16] Bacri, J. C., Cebers, A. O., \& Perzynski, R. (1994). Behavior of a magnetic fluid microdrop in a rotating magnetic field. Physical review letters, 72(17), 2705.

[17] Ezzaier, H., Alves Marins, J., Razvin, I, AbbasM., Ben Haj Amara, A., Zubarev A., Kuzhir P. Two-stage kinetics of field-induced aggregation of medium-sized magnetic nanoparticles. The Journal of Chemical Physics, 2017, 146(11) 114902

[18] L. D. Landau and E. M. Lifshitz, Statistical Physics, 3rd ed., Vol. 1 (Pergamon, Oxford, 1980). 
[19] A.Yu.Zubarev, A.O.Ivanov. Kinetics of a magnetic fluid phase separation induced by an external magnetic field. (1997) Phys. Rev. E., Vol. 55. 7192-7202.

[20] Levich, B, Physicochemical Hydrodynamics, Englewood Cliffs, N.J., Prentice-Hall, 1962 